\title{
Simplifying the Execution of HepatoPac MetID Experiments: Metabolite Profile and Intrinsic Clearance Comparisons ${ }^{\mathbb{}}$
}

\author{
T. Eric Ballard, ${ }^{1}$ N. Kratochwil, Loretta M. Cox, Mark A. Moen, F. Klammers, A. Ekiciler, \\ A. Goetschi, and I. Walter
}

Pharmacokinetics, Dynamics and Metabolism, Pfizer, Inc., Groton, Connecticut (T.E.B., L.M.C., M.A.M.) and Drug Disposition and Safety, Roche Pharmaceutical Research and Early Development, Roche Innovation Center, Basel, Switzerland (N.K., F.K., A.E., A.G., I.W.)

Received March 16, 2020; accepted June 12, 2020

\section{ABSTRACT}

The HepatoPac micropatterned coculture (MPCC) hepatocyte system has been shown to be an effective tool to investigate the qualitative human and preclinical species' metabolite profiles of new drug candidates. However, additional improvements to the overall study conditions and execution, layout, and human-donor count could be made. To that end, we have evaluated several ways to increase the amount of data one can generate per MPCC plate and how to more efficiently execute a MPCC study for the purpose of metabolite generation. Herein, we compare a set of compounds using single- and 10-donor pooled human MPCC hepatocytes. Intrinsic clearance and mean metabolic activities assessed by diverse enzyme markers were comparable between the singleand 10-donor pool. We have confirmed that the generated metabolite profiles were indistinguishable between the single- and 10-donor pool and also that rat MPCC can be performed at $400 \mu \mathrm{l}$ media volume, which greatly simplifies study execution. Additional tips for successful study execution are also described.

\section{SIGNIFICANCE STATEMENT}

When using the HepatoPac micropatterned coculture (MPCC) system, sometimes simple experimental condition variables or problematic plate designs can hamper productive study execution. We evaluated conditions to increase the amount of data one can generate per MPCC plate and, perhaps more importantly, execute that study more efficiently with less likelihood of error. We describe some of our key learnings, provide an examination of enzyme activity levels and clearance values, and provide some recommendations to simplify the execution of a HepatoPac experiment.

\section{Introduction}

Intrinsic clearance estimates and biotransformation studies of small molecules are an essential part of the drug discovery process (Plant, 2004; Pelkonen and Raunio, 2005; Kerns and Di, 2008). In the previous decades, liver preparations of microsomes, cytosol, S9 fraction, and, later, cryopreserved suspended hepatocytes proved amply useful at providing actionable metabolism information to progress programs forward (Plant, 2004; Pelkonen and Raunio, 2005; Dalvie et al., 2009). Chemists have become increasingly skilled at designing small molecules that are more metabolically stable, thereby driving clearance values ever lower. Very low-clearance compounds make the accurate measurement of intrinsic clearance and biotransformation studies increasingly difficult (Kerns and Di, 2008; Di and Obach, 2015; Hutzler et al., 2015). Over the past several years, several methods and products have risen to meet this challenge by prolonging the exposure of compounds (and their metabolites) to fresh hepatocytes through a relay or extending the stability of hepatocytes and allowing compounds to be incubated for many days and weeks without significant loss of enzyme activity (Khetani and Bhatia, 2008; Dash et al., 2009; Wang et al., 2010; Chan

${ }^{1}$ Current affiliation: Global Drug Metabolism and Pharmacokinetics, Takeda Pharmaceuticals Co., Cambridge, Massachusetts.

https://doi.org/10.1124/dmd.120.000013.

$\$$ This article has supplemental material available at dmd.aspetjournals.org. et al., 2013; Ballard et al., 2014, 2016; Bonn et al., 2016; Hultman et al., 2016; Burton et al., 2018).

In this study, we sought to investigate a few important questions regarding the HepatoPac system and to provide some clarity on the execution of metabolite identification (ID) studies in the HepatoPac system through the sharing of some of our solutions to problems we have encountered. We specifically sought to 1) compare the effect of media volume on metabolite profiles in rat micropatterned cocultured (MPCC) hepatocytes and 2) compare metabolite profiles and intrinsic clearance values of single-donor MPCC hepatocytes to a 10-donor pool of human MPCC hepatocytes. We sought to use a 10-donor pool to maximize the available incubations per plate/experiment, investigate future opportunities to create higher overall enzyme activity pools, and protect against single-donor interindividual variability in the HepatoPac system. For scientists working with rat MPCC and the multispecies HepatoPac plates, it is a common frustration to have to treat the rat MPCC hepatocyte wells differently than all the other species (recommended rat media volume is $300 \mu \mathrm{l}$ vs. $400 \mu \mathrm{l}$ for all other species), which can lead to complicated dosing and sampling regimes. So, to simplify experimental execution, we were interested in testing whether the media volume differences for rat MPCC hepatocytes resulted in any detectable differences in the metabolite profiles of our test compounds (Fig. 1). The test set was chosen to represent moderate- to low-clearance compounds with single and complex/multistep metabolic pathways across several

ABBREVIATIONS: $\mathrm{AO}$, aldehyde oxidase; $\mathrm{Cl}_{\text {int }}$, intrinsic clearance; ID, identification; LC, liquid chromatography; MPCC, micropatterned coculture; MS, mass spectrometry; UGT, urinidine 5'-diphospho-glucuronosyltransferase; UHPLC, ultra-high-pressure LC. 
<smiles>CCCNC(=O)OC1CCN(c2nncc3cc(OC)c(OC)cc23)CC1</smiles>

carbazeran<smiles>CCN(CC)CCNC(=O)c1c(C)[nH]c(/C=C2\C(=O)Nc3ccc(F)cc32)c1C</smiles>

sunitinib<smiles>CCCCNC(=O)NS(=O)(=O)c1ccc(C)cc1</smiles>

tolbutamide<smiles>CN1CCN(CCCN2c3ccccc3Sc3ccc(C(F)(F)F)cc32)CC1</smiles>

trifluoperazine<smiles>C=CC1CC2CN1CC2[C@H](O)c1ccnc2ccc(OC)cc12</smiles>

quinidine

Fig. 1. Set of test compounds to be used in this analysis.

enzyme classes as well as to complement our previous research with these compounds (Ballard et al., 2016).

The multispecies MPCC plate design could also be further simplified and made to accommodate a greater variety of species or testable experimental conditions if the three individual human-donor columns were eliminated in favor of a 10-donor human MPCC hepatocyte pool. A benefit of consolidating the three individual human donors into one column is that this would then allow for the fibroblast controls to be included on the same plate. To that end, we examined the intrinsic clearances of our set of test compounds in both single- and 10-donor MPCCs and also determined the enzyme activity levels of the singleand 10-donor MPCC systems using probe substrates for 13 common drug-metabolizing enzymes.

\section{Materials and Methods}

Materials. Carbazeran and quinidine were obtained from Pfizer Global Material Management (Groton, CT). Sunitinib was purchased from AK Scientific (Union City, CA). Tolbutamide and trifluoperazine were purchased from Sigma Aldrich (St. Louis, MO). MPCC hepatocyte 24-well plates containing humandonor VNL (female), pooled 10-donor human YFA (mixed-sex), rat donor, and mouse fibroblast controls were provided by Ascendance Biotechnology (Medford, MA - now BioIVT, Westbury, NY). The MPCCs were purchased from Ascendance Biotechnology and treated as previously described prior to shipment (Ballard et al., 2016). For performing biotransformation metabolite ID experiments, MPCC hepatocytes were plated in a 1:3 ratio of fibroblasts:hepatocytes (with $\sim 25 \mathrm{k}$ hepatocytes/well) in a 24-well plate. Ninety-six-well plates containing MPCC hepatocytes in a 1:3 ratio with 3200 hepatocytes/well were used for intrinsic clearance experiments (donor specification sheets provided by the supplier). A total hepatocyte protein content of $0.005 \mathrm{mg} \mathrm{ml}^{-1}(0.0032 \mathrm{mg}$ per well) was therefore applied for the 96-well HepatoPac format as described previously (Kratochwil et al., 2017). Fibroblast controls were included on the same plate for the biotransformation metabolite ID experiments (mouse and human), whereas separate fibroblast control plates were used for the intrinsic clearance experiments (mouse only). Unless stated otherwise, all other solvents and compounds were purchased from ThermoFisher Scientific (Waltham, MA).

Biotransformation Metabolite ID Using MPCC Hepatocyte Plates. Studies were adapted from previously described methods (Ballard et al., 2016). Briefly, 24-well MPCC plates were shipped to Pfizer, fresh medium was applied, and cultures were kept at $37^{\circ} \mathrm{C}$ with $90 \% \mathrm{O}_{2} / 10 \% \mathrm{CO}_{2}$ and $95 \%$ relative humidity for 2 days. MPCCs were changed to serum-free medium 2 hours prior to compound treatment. The MPCC hepatocytes (and on-plate fibroblast controls) were incubated with test compounds $\left(37^{\circ} \mathrm{C}\right.$ with $90 \% \quad \mathrm{O}_{2} / 10 \% \quad \mathrm{CO}_{2}$ and $95 \%$ relative humidity) at a final concentration of $10 \mu \mathrm{M}$ [chosen to provide quality UV and mass spectrometry (MS) data] in treatment media (DMSO final concentration $\approx 0.03 \%$ ) with a final volume of $400 \mu \mathrm{l}$ (with the exception of rat hepatocytes at $300 \mu \mathrm{l}$ for the comparison study). At 0,48 , and 168 hours, the culture medium was removed from respective wells and transferred to $15-\mathrm{ml}$ vials.
Time 0 samples were supernatant-only based on the sampling protocol, and proteins were precipitated with $1600 \mu \mathrm{l}$ of acetonitrile. For the 48- and 168-hour time points, the wells were transferred to $15-\mathrm{ml}$ vials, then the wells were washed with $2 \times 800 \mu$ l of acetonitrile (scraping the bottom of the wells with the pipet tips to detach cells), and the washes were pooled with the corresponding precipitated samples. The samples were mixed on a vortex mixer and then centrifuged $(1280 g$, 5 minutes), and the supernatants were transferred to new 15-ml conical glass tubes. The supernatants were dried in a Genevac (Genevac Inc, Valley Cottage, NY) evaporative centrifuge, and the resulting residues were reconstituted in $100 \mu \mathrm{l}$ of $95 \%$ water $(0.15 \%$ formic acid $) / 5 \%$ acetonitrile $(300-\mu l$ rat time point samples were reconstituted into $75 \mu \mathrm{l}$ with same $95 \% / 5 \%$ proportions to normalize the resuspension concentrations), centrifuged (1280g, 5 minute), and analyzed by liquid chromatography (LC) MS. All compounds were incubated in the MPCC hepatocytes with the same time point collections, regardless of known or expected clearance rates (human or rat), based on previous studies in HepatoPac and hepatocyte relay systems (Ballard et al., 2014, 2016). For a more detailed description on the execution of the biotransformation metabolite ID study, please see the expanded section (vide infra).

Ultra-High-Pressure LC Tandem Mass Spectrometry Analysis for Metabolite ID Profiling. Mass spectrometric conditions were adapted from previously described methods (Ballard et al., 2016). Briefly, reconstituted samples were analyzed by UHPLC-UV-MS operated in positive or negative ion mode (negative mode only used for detection of tolbutamide $N$-dealkylation product) using an Orbitrap Elite mass spectrometer. For UHPLC-UV-MS analysis, the capillary temperature was set at $275^{\circ} \mathrm{C}$, the source potential was $3500 \mathrm{~V}$ (used same source potential for both positive and negative mode scanning), and the source heater was set at $425^{\circ} \mathrm{C}$. The mass spectrometer was operated in a datadependent scanning mode to $\mathrm{MS}^{3}$ with dynamic exclusion enabled (repeat count: 1; repeat duration: 5.0 seconds; list size: 500; exclusion duration: 1.5 seconds). The normalized collision energy for the data-dependent scans was 35\%. Other potentials were adjusted as necessary to get optimal ionization and fragmentation of the parent compound. UV absorption spectra were obtained by an in-line Accela photodiode array detector. A Kinetex EVO C18 $100 \AA$ A column $(2.1 \times 150$ $\mathrm{mm}, 1.7 \mu \mathrm{m}$; Phenomenex, Torrance, $\mathrm{CA}$ ) was used with a flow rate of $0.4 \mathrm{ml} / \mathrm{min}$ at $45^{\circ} \mathrm{C}$ (except for quinidine: flow rate of $0.3 \mathrm{ml} / \mathrm{min}$ ). Mobile phase A was comprised of $0.1 \%$ formic acid, and mobile phase B was comprised of acetonitrile. For carbazeran, sunitinib, and tolbutamide, the gradient system used was as follows: initially, $5 \% \mathrm{~B}$ was held for 0.8 minutes, and this was followed by a linear gradient to $50 \% \mathrm{~B}$ from 0.8 to 8.25 minutes, a second linear gradient to $95 \% \mathrm{~B}$ at $8.5-8.75$ minutes, a 0.25 -minute wash at $95 \% \mathrm{~B}$, a third linear gradient to $5 \% \mathrm{~B}$ at 9-9.2 minutes, and finally a 0.8 -minute reequilibration period at $5 \%$ B. For trifluoperazine, the gradient system used was as follows: initially, $5 \%$ B was held for 0.8 minutes, and this was followed by a linear gradient to $95 \% \mathrm{~B}$ from 0.8 to 8.25 minutes, a 0.75 -minute wash at $95 \% \mathrm{~B}$, a third linear gradient to $5 \% \mathrm{~B}$ at 9-9.2 minutes, and finally a 0.8 -minute reequilibration period at $5 \%$ B. For quinidine, the flow rate was $0.3 \mathrm{ml} / \mathrm{min}$, and the gradient system used was as follows: initially, $5 \% \mathrm{~B}$ was held for 0.8 minutes, and this was followed by a linear gradient to $40 \% \mathrm{~B}$ from 0.8 to 8.25 minutes, a second linear gradient to $95 \% \mathrm{~B}$ at 8.5-8.75 minutes, a 0.25 -minute wash at $95 \% \mathrm{~B}$, a third linear gradient to $5 \% \mathrm{~B}$ at 
9-9.2 minutes, and finally a 0.8 -minute reequilibration period at $5 \% \mathrm{~B}$. Injections of $10 \mu \mathrm{l}$ were made by a CTC Prep and Load autosampler (CTC Analytics, Zwingen, Switzerland).

Intrinsic Clearance Determinations and Metabolic Activity Profiling. Studies were adapted from previously described methods (Kratochwil et al., 2017). Briefly, after a recovery of 48 hours at $37^{\circ} \mathrm{C}$ and $10 \% \mathrm{CO}_{2}$, the HepatoPac plates were washed once with application medium ( $64 \mu \mathrm{l}$ serum-free Dulbecco's modified Eagle's medium specially formulated for HepatoPac cultures), and then fresh media $(64 \mu \mathrm{l})$ was preincubated for 2 hours at $37^{\circ} \mathrm{C}$ and $10 \% \mathrm{CO}_{2}$ before adding the compounds. For clearance determinations, quinidine, sunitinib, and tolbutamide were tested at a concentration of $1 \mu \mathrm{M}$, and trifluoperazine was tested at a concentration of $0.3 \mu \mathrm{M}$ and carbazeran at concentrations of $0.3,1$, and $3 \mu \mathrm{M}$, respectively [no concentration dependency of intrinsic clearance $\left(\mathrm{Cl}_{\text {int }}\right)$ was observed for carbazeran in MPCC hepaptocyes (single- or 10-donor) in contrast to suspension hepatocytes (details are given in the Supplemental Material)]. For metabolic activity determination, the following enzyme substrates were added to HepatoPac plates and incubated at $5 \% \mathrm{CO}_{2}$ atmosphere and at $37^{\circ} \mathrm{C}$ : benzydamine (flavin-containing monooxygenase) at $1 \mu \mathrm{M}$; midazolam (CYP3A4) and dextromethorphan (CYP2D6) at $5 \mu \mathrm{M}$; 7-hydroxycoumarin [sulfotransferase and urinidine 5 '-diphospho-glucuronosyltransferase (UGT)], diclofenac (CYP2C9), tacrine (CYP1A2), daunorubicin (aldo-keto reductase), and $\mathrm{O}_{6}$-benzyl guanine [aldehyde oxidase (AO)] at $10 \mu \mathrm{M}$; bupropion (CYP2B6), amodiaquine (CYP2C8), and sulfamethazine ( $N$-acetyltransferase) at $20 \mu \mathrm{M}$; and $\mathrm{SN}-38$ (UGT1A1) at $50 \mu \mathrm{M}$, respectively. The final DMSO content in the medium was $0.1 \%$ DMSO. At defined time points, samples were drawn and quenched with acetonitrile (volume ratio 1:2) containing an internal standard (oxazepam). Samples were then cooled, centrifuged $(6000 \mathrm{~g}, 10$ minutes), and quantified by using LC with tandem MS.

High-Pressure LC-Mass Spectrometry Analysis for Intrinsic Clearance and Metabolic Activity. Mass spectrometric conditions were adapted from previously described methods (Kratochwil et al., 2017). Briefly, LC with tandem MS was used for the quantification of the compounds. The high-pressure LC system consisted of 30AD Shimadzu pumps. The analytical columns used were a Phenomenex Synergi Polar-RP C18 $(5 \mathrm{~cm} \times 1 \mathrm{~mm}$; $3.5 \mu \mathrm{m})$ for $7-$ hydroxycoumarin and a Supelco Ascentis Express C18 $(2 \mathrm{~cm} \times 2.1 \mathrm{~mm} ; 2.7$ $\mu \mathrm{m})$ at $60^{\circ} \mathrm{C}$ for the other analytes. A Q6500+ Sciex mass spectrometer equipped with a TurboIonSpray source (IonSpray Voltage $5500 \mathrm{~V}$ in positive mode, $-4500 \mathrm{~V}$ in negative mode) and an HTS CTC Prep and Load autosampler were used. Mobile phase A was 0.5\% formic acid in water:methanol 95:5, mobile phase B was acetonitrile. Aliquots $(1 \mu \mathrm{l})$ of the diluted sample solutions were injected and transferred to the analytical column at a flow rate of $600 \mu \mathrm{l} / \mathrm{min}$. To elute the compounds, a high-pressure linear gradient from $0 \%$ to $95 \%$ B in 40 seconds was applied. Total run time was 1.6 minutes.

Data Analysis for Intrinsic Clearance and Metabolic Activity. Data were analyzed as described previously using quadratic regression with $1 / \mathrm{x}^{2}$ weighting on peak area ratios (Kratochwil et al., 2017). The precision and accuracy of the standard and quality control samples were between $80 \%$ and $120 \%$. Analyst software (Version 1.6.2; Sciex, Framingham) was employed for data processing. For determination of the intrinsic clearance, pharmacokinetic modeling was applied in the Monolix software (Version 4.33; Lixoft - Incuballiance, Orsay, France) by using a nonlinear mixed-effects approach to in vitro single-time concentration profiles. The statistical unit was the well, and interwell variability on the derived parameters was explored. The details for the intrinsic clearance determination are described elsewhere (Kratochwil et al., 2017). To calculate the metabolic rates, a linear fit was made to the determined concentrations of the metabolites plotted against time. The linear rate was then normalized by the protein content of the cells to derive the metabolite formation rate (picomoles per minute per milligram protein). To derive the metabolic activity index (microliters per minute per milligram protein), the derived metabolite formation rates were further divided by the substrate concentration used in the incubation (Supplemental Table 2). Intrinsic clearance values and metabolic rates were also determined in mouse embryonic 3T3 fibroblast-only control plates, and the derived intrinsic clearance and metabolic rates were corrected as described previously (Kratochwil et al., 2017) (Supplemental Table 3).

Expanded Biotransformation Metabolite ID Protocol. For comparison of the single human VNL donor and the 10-donor pooled MPCC hepatocytes, two 24-well plates were used with the first plate containing the single human-donor MPCC and the second containing the 10-donor pooled MPCC hepatocytes. Plates were divided in half with the MPCC on one side and the stromal only controls on the other (Supplemental Excel File - Plate Map). Columns 1 and 6 were used as control wells, and test compounds were dosed in columns 2-5. Rows 1 and 4 were used for the time 0/day 2 samples, whereas rows 2 and 3 were used for day 7 samples. By having the earliest time points and controls located on the outside wells, loss of volume due to evaporation in the later time points was mitigated (despite 95\% humidity, evaporation was still noticeable after 7 days).

To streamline compound and sample manipulation, a dosing plate was prepared containing $2 \times$ concentrations of test compounds in media. With a variety of compounds being tested on multiple plates, implementing a dosing plate in the same format as the test plates allowed for a smoother workflow and reduced the chance of sample-handling error. After 2-hour incubation with the serum-free media, the media was removed, and then half of the media volume (200 $\mu 1)$ was added to the wells prior to dosing with test compound. One hundred sixtyeight-hour wells were then dosed first from the dosing plate followed by the 0/48hour wells. Supernatant samples were removed immediately from the 0/48-hour wells and transferred to conical tubes containing $4 \times$ volumes of acetonitrile. Media $(200 \mu \mathrm{l})$ was then immediately added back to the $0 / 48$-hour wells and then redosed with test compound from the dosing plate to be used as the 48-hour samples. Forty-eight- and 168-hour time points were sampled by removing the entire supernatant volume of the respective wells and transferring to conical vials. Acetonitrile $(2 \times$ volume) was then added to the wells, and the bottom of the plates were exfoliated with pipet tips. The resulting suspensions were then pooled with the corresponding supernatants. Wells were washed a second time with acetonitrile $(2 \times$ volume) and pooled with the corresponding suspensions. When taking the 48-hour time point, blank media were added back to the sampled wells to maintain humidity levels in the plate.

Wells containing rat hepatocytes were treated similarly as described above. Wells only containing $300 \mu \mathrm{l}$ of media were prepared in the same manner as wells with $400 \mu \mathrm{l}$ of media, except after dosing the plate, $100 \mu \mathrm{l}$ of sample was removed from each rat hepatocyte well and discarded (leaving $300 \mu \mathrm{l}$ ). The human MPCC time point-sampling protocol outlined above was used for the rat MPCC studies.

\section{Results}

Rat Media Volume Assessment. The test set of compounds was incubated at $10 \mu \mathrm{M}$ in rat MPCC hepatocytes for 168 hours, taking 0-, 48-, and 168-hour time points, respectively. Using the 168-hour time point as representative, metabolite ID profiles for each compound were compared and were indistinguishable when run at the recommended $300-\mu 1$ volume versus the desired $400-\mu 1$ volume (Figs. 2 and 3; Supplemental Figs. 1-9).

Single Human-Donor VNL versus 10-Donor Pool MPCC Assessment of MetID Profile and Clearance Rates. The test set of compounds were incubated at $10 \mu \mathrm{M}$ in single- and 10-donor pooled human MPCC hepatocytes for 168 hours taking 0-, 48-, and 168-hour time points, respectively. The 10-donor pool was prepared by BioIVT independent of this investigation, and so the VNL single donor was not specifically included as one of the donors within the pool. The VNL donor was used in this investigation from previous experience (Ballard et al., 2016), and there was no specific selection criteria imposed beyond VNL representing average metabolic activity. Using the 168-hour time point as representative, metabolite ID profiles for each compound were compared and were indistinguishable between the single- and 10-donor incubations (Fig. 4; Supplemental Figs. 11-14). Intrinsic clearance values were determined at $1 \mu \mathrm{M}$ (trifluoperazine $0.3 \mu \mathrm{M}$ and carbazeran 0.3-3 $\mu \mathrm{M}$ ) with time points taken up to 96 hours (Supplemental Fig. 10; Supplemental Table 4; Table 1). Similar $\mathrm{Cl}_{\text {int }}$ values were obtained for quinidine, sunitinib, trifluoperazine, and carbazeran, whereas tolbutamide displayed a 2.7-fold lower clearance value in the 10-donor pool.

Metabolic Activity Assessment of Single Human-Donor VNL versus 10-Donor Pool MPCC. Thirteen diverse phase I and phase II enzyme markers were used to compare metabolic activity of the singledonor versus 10-donor MPCC hepatocytes. In general, similar mean metabolic activities were found for the single VNL donor and the 


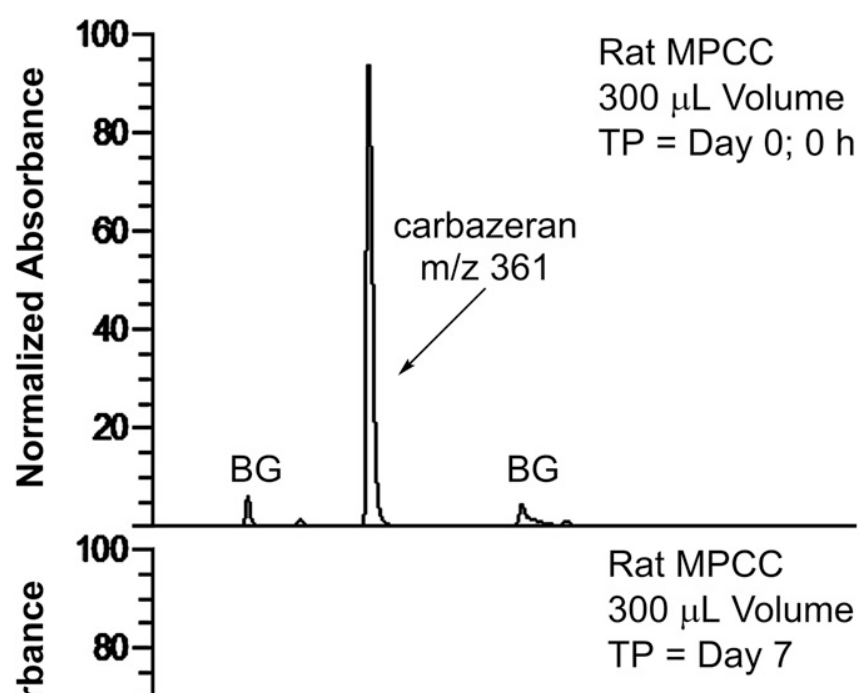

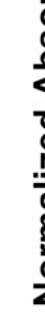

政
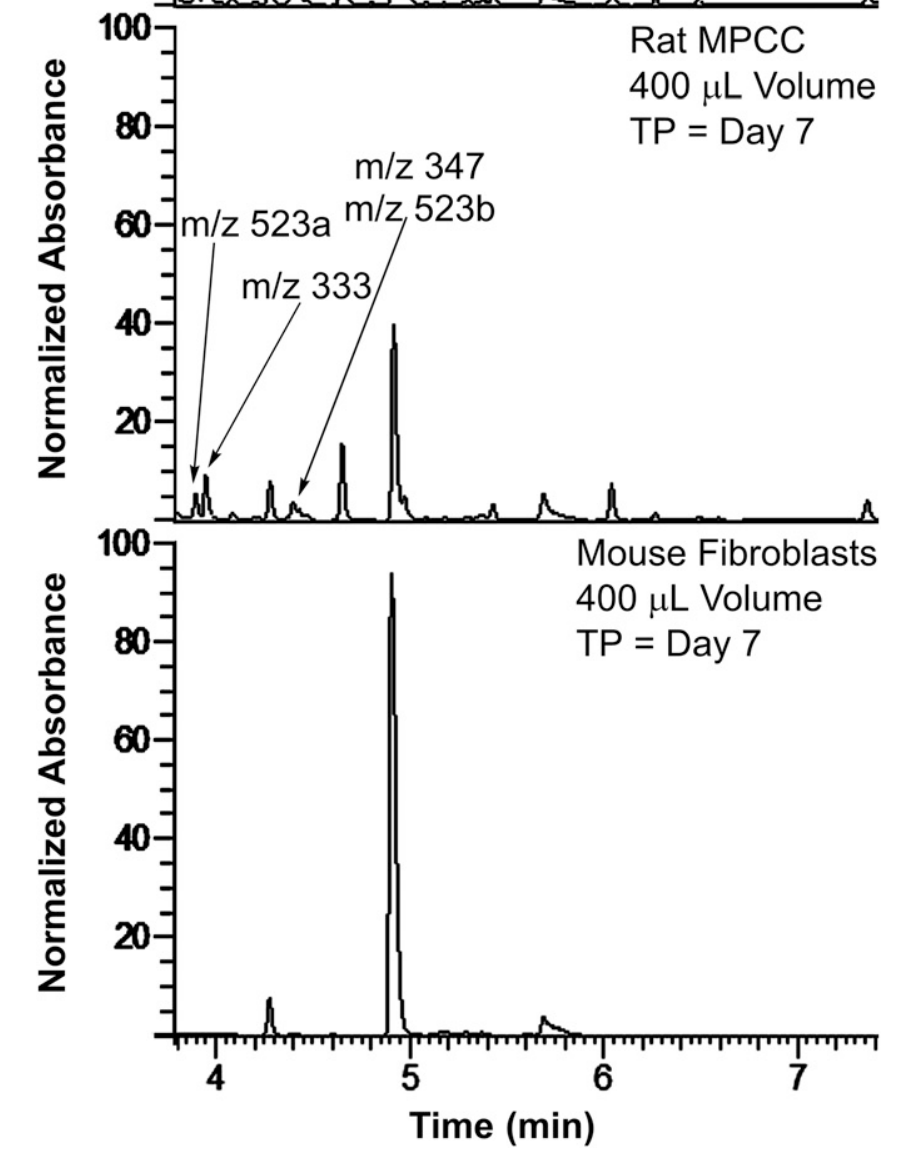

Fig. 2. Representative UV-UHPLC chromatograms of carbazeran metabolism in rat MPCC hepatocytes. BG, background; TP, time point. 10-donor pool with average metabolic activity index values of 58 and 37 , respectively. The single VNL donor displayed an average 1.6-fold higher activity across of the enzyme marker studied (Fig. 5; Supplemental Table 1). Notable differences were $N$-acetyltransferase with 3-fold higher activity in the single donor, whereas CYP2B6 was $>10$-fold lower in the single donor.

\section{Discussion}

Our overall goal in this investigation was to streamline the execution of a biotransformation metabolite identification experiment using a multispecies MPCC HepatoPac plate and confirm that a 10-donor human MPCC HepatoPac plate was equivalent to or better than a single donor for both metabolite identification and intrinsic clearance determinations. We sought to use a 10-donor pool to maximize the available incubations per plate/experiment and protect against single-donor interindividual variability in the HepatoPac system. Our first evaluation began with assessing the effects of incubations volumes on the rat MPCC hepatocyte metabolite profiles with a test set of compounds. The compounds were incubated at $10-\mu \mathrm{M}$ final concentration in either 300 or $400 \mu \mathrm{l}$ media volume, and samples were removed for analysis at initiation of the experiment ( $\mathrm{T}=0$ hours) and at 48 and 168 hours of incubation at $37^{\circ} \mathrm{C}$. Regardless of time point, metabolite profiles for the test set of compounds at the recommended 300- $\mu$ l media volume for rat MPCC hepatocytes were indistinguishable from the 400- $\mu$ l incubations (Fig. 2; Supplemental Figs. 1-8). This result, although on a limited test set, provides evidence that the rat and the multispecies MPCC (that includes rat MPCC) HepatoPac plate can be simplified to use $400 \mu 1$ of media volume across the entire plate, thus greatly decreasing the chance for dosing error and providing an easier execution of the experiment. Carbazeran, as a representative example, displayed an equal production of metabolites resulting from multiple metabolic pathways: oxidative demethylation $(\mathrm{m} / \mathrm{z} 333$ and 347), AO $(\mathrm{m} / \mathrm{z}, 377)$, direct conjugations $(\mathrm{m} / \mathrm{z}, 523 \mathrm{~b}$ and $\mathrm{m} / \mathrm{z}, 537)$, and secondary metabolites $(\mathrm{m} / \mathrm{z}, 349,427$, and 523a) (Figs. 2 and 3). Tolbutamide metabolism in rat forms a urea dealkylation metabolite that is not formed in human, and the formation of this metabolite was also unaffected by the incubation media volume (Supplemental Fig. 9) (Gee et al., 1984; Back and Orme, 1989). Clearance rates of the test compounds were not assessed in rat MPCC hepatocytes at either volume. No metabolism was detected for any of the test compounds in mouse fibroblast coculture cells at either volume tested (Fig. 2; Supplemental Figs. 1-4).

After our evaluation of the rat media volume, we sought to assess the ability to simplify the HepatoPac plate design by using a 10-donor human pool versus using three separate donors on a single plate. Throughout discovery absorption, distribution, metabolism, and excretion scientific investigations, donor pools were used for nearly all preclinical and human (usually a pool of 10+ mixed-sex) liver subcellular fractions (especially hepatocytes) in an effort to maximize enzymatic activity and protect against interindividual variability. A follow-on output of this research was to provide evidence that a platable pool of 10 donors could, potentially, be used in place of the three individual hepatocyte donors. Generating a pool of platable hepatocytes and ensuring that all hepatocyte donors are equally represented in the MPCC are not simple tasks and are still actively under investigation. However, the activity of the 10 individual donors was assessed in suspension across a number of enzymes, and their mean activity was in agreement with the activity of the pooled hepatocyte MPCCs, suggesting equivalent representation of each donor (Heyward et al., 2016).

To evaluate the metabolite profile differences that could arise, the set of compounds was incubated at $10 \mu \mathrm{M}$ in single VNL- and 10-donor 
<smiles></smiles>

$\mathrm{m} / \mathrm{z} 427$<smiles>CCNC(=O)OC1CCN(c2nncc3cc(O)c(OC)cc23)CC1</smiles>

$\mathrm{m} / \mathrm{z} 347$<smiles>[R16]c1cnc(N2CCC(OC(=O)NCC)CC2)c2cc(OC)c(O[C@@H]3O[C@H](C(=O)O)[C@@H](O)[C@H](O)[C@H]3O)cc12</smiles>

$\mathrm{m} / \mathrm{z}$ 523a<smiles>CCNC(=O)OC1CCN(c2n[n+]([C@H]3OC(C(=O)O)[C@@H](O)[C@H](O)[C@H]3O)cc3cc(OC)c(OC)cc23)CC1</smiles>

$\mathrm{m} / \mathrm{z} 537$
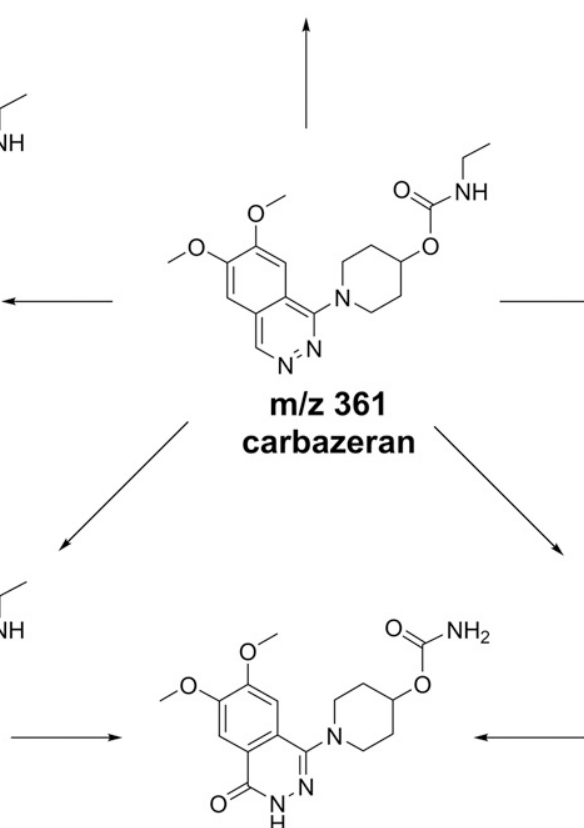

$\mathrm{m} / \mathrm{z} 349$<smiles>CCNC(=O)OC1CCN(c2nnc([C@H]3OC(CO)[C@@H](O)[C@H](O)[C@H]3O)c3cc(OC)c(OC)cc23)CC1</smiles>

$\mathrm{m} / \mathrm{z} 523 \mathrm{~b}$
Fig. 3. Metabolic scheme of carbazeran in rat and human MPCC hepatocytes. Position of catechol demethylation and subsequent conjugation not known. 4-Position oxo-carbazeran and des-ethyl metabolites $(\mathrm{m} / \mathrm{z} 333, \mathrm{~m} / \mathrm{z} 349$ and $\mathrm{m} / \mathrm{z} 377)$ and $3-\mathrm{N}$-conjugate metabolite structures $(\mathrm{m} / \mathrm{z}, 523 \mathrm{~b}$ and $\mathrm{m} / \mathrm{z} 537)$ inferred from literature (Kaye et al., 1984)<smiles>COc1cc2cnnc(N3CCC(OC(N)=O)CC3)c2cc1OC</smiles>

$\mathrm{m} / \mathrm{z} 333$
MPCC hepatocytes for 168 hours, taking time points at 0,48 , and 168 hours. In parallel experiments, $\mathrm{Cl}_{\text {int }}$ was also measured for each compound over the course of several hours to days (depending on the rate of clearance) in both the single- and 10-donor MPCC hepatocytes.

As a whole, $\mathrm{Cl}_{\text {int }}$ for the set of compounds was comparable between the single- and 10-donor MPCC hepatocyte incubations. $\mathrm{Cl}_{\text {int }}$ values (microliters per minute per milligram protein) for quinidine, sunitinib, trifluoperazine, and carbazeran were similar between the single- and 10-donor pool (Table 1), which also provides evidence that UGT1A1 (trifluoperazine), CYP3A (quinidine and sunitinib), and AO (carbazeran) have equivalent activities between the two systems (further evaluated below). Tolbutamide, a CYP2C9 substrate, displayed a 2.7fold higher clearance in the single-donor MPCC, which can be attributed to donor variability (Lombardo et al., 2013; Jones et al., 2015). Although the clearance for tolbutamide was higher in the single-donor MPCC, the metabolite profile was indistinguishable between the single- and 10donor MPCC human hepatocytes (Fig. 4; Supplemental Fig. 11). To that end, the metabolite profiles for quinidine, sunitinib and trifluoperazine, carbazeran, and tolbutamide in the single- and 10-donor MPCC hepatocytes were qualitatively indistinguishable from each other at all time points (Supplemental Figs. 12-14).

As a follow-up to the $\mathrm{Cl}_{\text {int }}$ and metabolite profile assessments of our test set of compounds, we also investigated the enzymatic activity of the single- and 10-donor MPCC hepatocytes using 13 diverse enzyme markers. Metabolite formation was measured in both systems for up to 6 hours. Similar mean metabolic activities were found for the single VNL donor and the 10-donor pool across the studied enzyme markers. On average, the single donor displayed 1.6-fold higher activity
(Fig. 5; Supplemental Table 1) compared with the 10-donor pool. $\mathrm{N}$-acetyl transferase was shown to have 3 -fold higher activity in the single donor, and CYP2B6 was significantly less active as compared with the 10-donor pool. The higher 1.9-fold AO and 1.6-fold CYP2C9 activities using different small molecules as substrates (diclofenac and $\mathrm{O}_{6}$-benzyl guanine, respectively) for the metabolic activity determination are in agreement with the higher intrinsic clearance values for tolbutamide (CYP2C9 substrate) and carbazeran (AO substrate). The derived mean metabolic activity index compares enzyme activities across diverse phase I and II hepatic enzymes and can be used to assess donor variability and different in vitro liver systems. This index experiment is independent of the enzyme marker concentration used in the incubation to compare metabolic activity in MPCC hepatocyte or other in vitro liver systems across different test conditions (Supplemental Table 2).

\section{Conclusions and Recommendations}

We believe we have identified conditions and protocols that allow for a more facile execution of a MPCC hepatocyte experiment for metabolite identification that also can be designed to generate more data on a single plate. We have determined that the rat media volume can be $400 \mu \mathrm{l}$ to be consistent across the plate with the other MPCC species. Having one plate with two different sampling volumes added complexity and had the potential for mistakes during the dosing and sampling processes. By showing that the rat media volume no longer needs to be $300 \mu \mathrm{l}$, the dosing and sampling of the plate is now far simpler and less prone to sampling error. The metabolite profiles of our test set of compounds were indistinguishable in rat MPCC between the two 


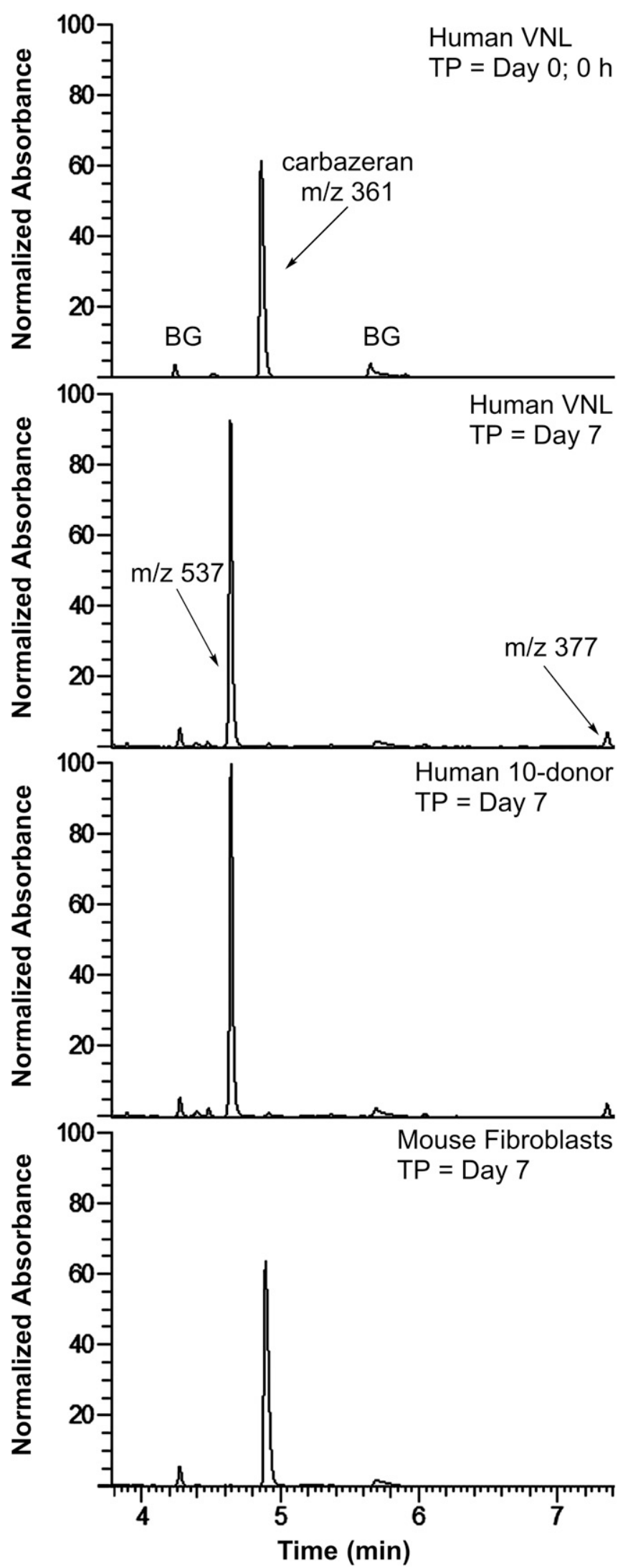

Fig. 4. Representative UV-UHPLC chromatograms of carbazeran metabolism in single- and 10-donor MPCC hepatocytes. BG, background; TP, time point.
TABLE 1

Intrinsic clearance determinations for the test set of compounds

\begin{tabular}{lcccc}
\hline & & & $\mathrm{Cl}_{\text {int }}\left(\mu \mathrm{min}^{-1} \mathrm{mg}^{-1}\right.$ protein) \\
\cline { 4 - 5 } Compounds & $\begin{array}{c}\text { Enzyme } \\
\text { Markers }\end{array}$ & Conc. $(\mu \mathrm{M})$ & VNL Donor (RSE\%) & $\begin{array}{c}\text { Multiple Donor } \\
\text { Pool (RSE\%) }\end{array}$ \\
\hline Carbazeran & AO & $0.3-3$ & $79.9(4)$ & $57.5(4)$ \\
Sunitinib & CYP3A4 & 1 & $10.0(1)$ & $9.9(2)$ \\
Tolbutamide & CYP2C9 & 1 & $4.6(4)$ & $1.7(10)$ \\
Trifluoperazine & UGT1A4 & 0.3 & $25.8(4)$ & $24.8(2)$ \\
Quinidine & CYP3A4 & 1 & $13.6(4)$ & $13.5(4)$ \\
\hline
\end{tabular}

RSE\%, relative standard error.

volumes tested. In addition to this change, the implementation, and use of a cloned dosing plate at $2 \times$ drug concentration was also instrumental to the smooth execution of an MPCC hepatocyte metID experiment with less chance of sample-handling error.

With the use of the 10-donor human, a new plate design can be envisioned (Fig. 6) that combines human, monkey, dog, and rat on the same plate with their respective fibroblast controls. This design allows for the dog MPCC to be directly adjacent to the respective controls.

Similar metabolic activity was found for the single VNL donor and the 10-donor pool across studied enzyme markers. The observed difference in enzyme activities is likely due to donor variability. With the investigation of only one single- and 10-donor pooled human MPCC hepatocyte, our conclusion regarding the equivalence of the test systems is, however, limited, and further investigations of new donors will help to substantiate our findings.

The use of the 10-donor human MPCC pool protects against any seeding, plating, and activity discrepancies that may occur with a single donor and also protects against any interindividual variability. Additional research to identify higher average activity donors to maximize overall enzyme activities would aid in the production of more robust metabolite profiles for comparison with in vivo preclinical and clinical samples to support in vitro-in vivo correlation translation.

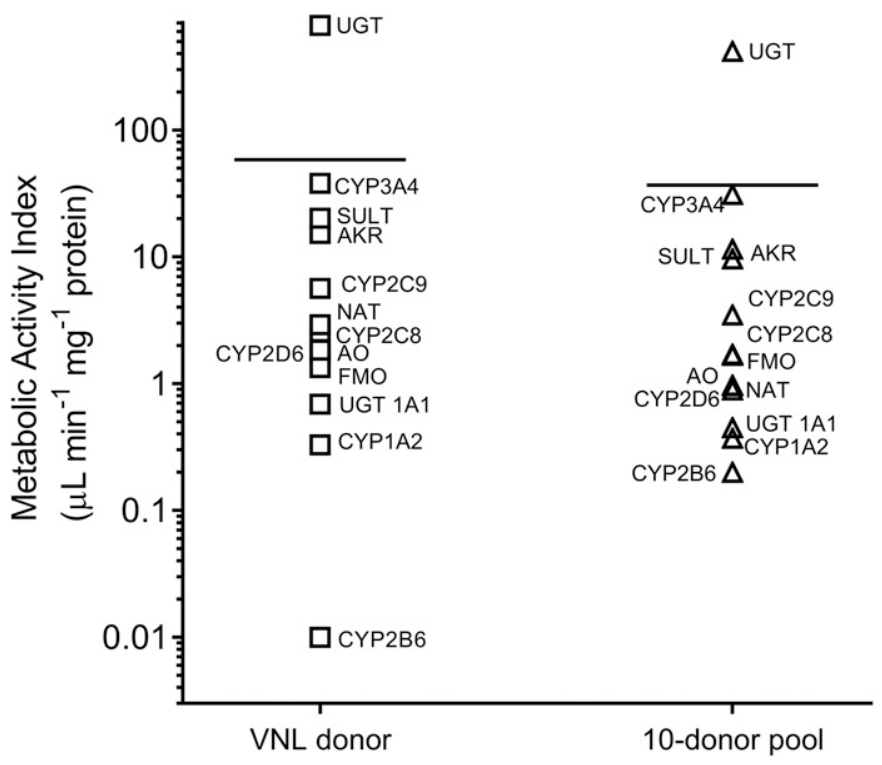

Fig. 5. Metabolic profiling of single-donor vs. 10-donor MPCC hepatocytes with selective enzyme substrates. Line represents the mean of the derived metabolic activity index values. Similar mean metabolic activities were found for the single VNL donor and the 10-donor pool with average values of 58 and 37, respectively. AKR, aldo-keto reductase; FMO, flavin-containing monooxygenase; NAT, $\mathrm{N}$-acetyltransferase; SULT, sulfotransferase. 
Time Points

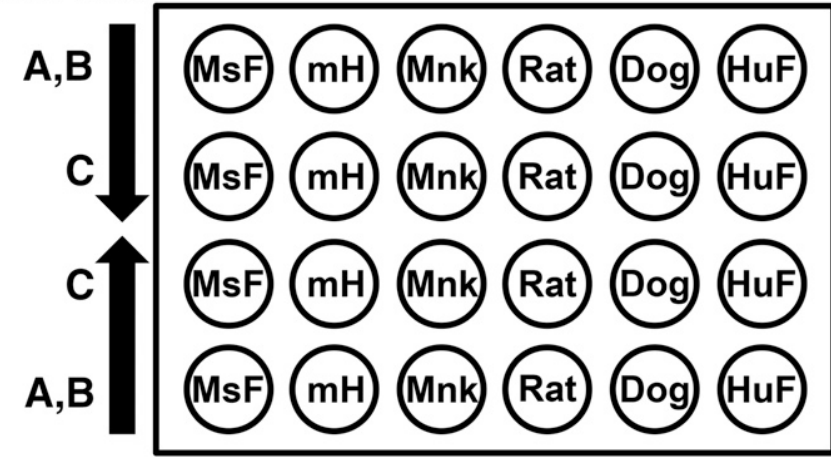

Fig. 6. Proposed multispecies MPCC hepatocyte plate including 10-donor human and fibroblast controls for all plated species. Time point $\mathrm{A}=$ day 0,0 -hour media removal; $\mathrm{B}=48$-hour media and cells; $\mathrm{C}=168$-hour media and cells. HuF, human fibroblast; $\mathrm{mH}$, mouse hepatocyte; MsF, mouse fibroblast; Mnk, monkey.

\section{Acknowledgments}

The authors would like to thank Stacy Krzyzewski, Onyi Irrechukwu, Jeannemarie Gaffney, and Jack McGeehan from Ascendance Biotechnologies/ BioIVT for supplying the MPCC plates and for helpful discussions.

\section{Authorship Contributions}

Participated in research design: Ballard, Kratochwil, Cox, Moen, Klammers. Conducted experiments: Cox, Moen, Klammers, Ekiciler, Goetschi, Walter. Performed data analysis: Ballard, Kratochwil, Klammers.

Wrote or contributed to the writing of the manuscript: Ballard, Kratochwil, Cox, Moen, Klammers, Ekiciler, Goetschi, Walter.

\section{References}

Back DJ and Orme ML (1989) Genetic factors influencing the metabolism of tolbutamide. Pharmacol Ther 44:147-155.

Ballard TE, Orozco CC, and Obach RS (2014) Generation of major human excretory and circulating drug metabolites using a hepatocyte relay method. Drug Metab Dispos 42:899-902.

Ballard TE, Wang S, Cox LM, Moen MA, Krzyzewski S, Ukairo O, and Obach RS (2016) Application of a micropatterned cocultured hepatocyte system to predict preclinical and humanspecific drug metabolism. Drug Metab Dispos 44:172-179.

Bonn B, Svanberg P, Janefeldt A, Hultman Ia, and Grime K (2016) Determination of human hepatocyte intrinsic clearance for slowly metabolized compounds: comparison of a primary hepatocyte/stromal cell co-culture with plated primary hepatocytes and HepaRG. Drug Metab Dispos 44:527-533.
Burton RD, Hieronymus T, Chamem T, Heim D, Anderson S, Zhu X, and Hutzler JM (2018) Assessment of the biotransformation of low-turnover drugs in the H $\mu$ REL human hepatocyte coculture model. Drug Metab Dispos 46:1617-1625.

Chan TS, Yu H, Moore A, Khetani SR, and Tweedie D (2013) Meeting the challenge of predicting hepatic clearance of compounds slowly metabolized by cytochrome P450 using a novel hepatocyte model, HepatoPac [published correction appears in Drug Metab Dispos (2014) 42:200; Drug Metab Dispos (2019) 47:58-66]. Drug Metab Dispos 41:2024-2032.

Dalvie D, Obach RS, Kang P, Prakash C, Loi CM, Hurst S, Nedderman A, Goulet L, Smith E, Bu HZ, et al. (2009) Assessment of three human in vitro systems in the generation of major human excretory and circulating metabolites. Chem Res Toxicol 22:357-368.

Dash A, Inman W, Hoffmaster K, Sevidal S, Kelly J, Obach RS, Griffith LG, and Tannenbaum SR (2009) Liver tissue engineering in the evaluation of drug safety. Expert Opin Drug Metab Toxicol 5:1159-1174

Di L and Obach RS (2015) Addressing the challenges of low clearance in drug research. AAPS J 17:352-357.

Gee SJ, Green CE, and Tyson CA (1984) Comparative metabolism of tolbutamide by isolated hepatocytes from rat, rabbit, dog, and squirrel monkey. Drug Metab Dispos 12:174-178

Heyward S, McCauley P, and Moeller T (2016) Utility of pooled cryoplateable LIVERPOOL ${ }^{\mathrm{TM}}$ in the in vitro determination of cytochrome P450 metabolism and induction. 20th North American ISSX Meeting: P184. Drug Metab Rev 48:28.

Hultman Ia, Vedin C, Abrahamsson A, Winiwarter S, and Darnell M (2016) Use of H $\mu$ REL human coculture system for prediction of intrinsic clearance and metabolite formation for slowly metabolized compounds. Mol Pharm 13:2796-2807.

Hutzler JM, Ring BJ, and Anderson SR (2015) Low-turnover drug molecules: a current challenge for drug metabolism scientists. Drug Metab Dispos 43:1917-1928.

Jones HMC, Chen Y, Gibson C, Heimbach T, Parrott N, Peters SA, Snoeys J, Upreti VV, Zheng $\mathrm{M}$, and Hall SD (2015) Physiologically based pharmacokinetic modeling in drug discovery and development: a pharmaceutical industry perspective. Clin Pharmacol Ther 97:247-262.

Kaye B, Offerman JL, Reid JL, Elliott HL, and Hillis WS (1984) A species difference in the presystemic metabolism of carbazeran in dog and man. Xenobiotica 14:935-945.

Kerns EH and Di L (2008) Drug-Like Properties: Concepts, Structure Design, and Methods: From ADME to Toxicity Optimization, Academic Press, Burlington, MA.

Khetani SR and Bhatia SN (2008) Microscale culture of human liver cells for drug development. Nat Biotechnol 26:120-126.

Kratochwil NA, Meille C, Fowler S, Klammers F, Ekiciler A, Molitor B, Simon S, Walter I, McGinnis C, Walther J, et al. (2017) Metabolic profiling of human long-term liver models and hepatic clearance predictions from in vitro data using nonlinear mixed-effects modeling. AAPS J 19:534-550.

Lombardo F, Waters NJ, Argikar UA, Dennehy MK, Zhan J, Gunduz M, Harriman SP, Berellini G, Liric Rajlic I, and Obach RS (2013) Comprehensive assessment of human pharmacokinetic prediction based on in vivo animal pharmacokinetic data, part 2: clearance. J Clin Pharmacol 53: $178-191$.

Pelkonen O and Raunio H (2005) In vitro screening of drug metabolism during drug development: can we trust the predictions? Expert Opin Drug Metab Toxicol 1:49-59.

Plant N (2004) Strategies for using in vitro screens in drug metabolism. Drug Discov Today 9 328-336.

Wang WW, Khetani SR, Krzyzewski S, Duignan DB, and Obach RS (2010) Assessment of a micropatterned hepatocyte coculture system to generate major human excretory and circulating drug metabolites. Drug Metab Dispos 38:1900-1905.

Address correspondence to: T. Eric Ballard, Global Drug Metabolism and Pharmacokinetics, Takeda Pharmaceuticals Co., 35 Landsdowne St., Cambridge, MA 02139. E-mail: eric.ballard@takeda.com 